\title{
Intelligent storage and washing integrated cabinet based on sine mechanism
}

\author{
$\mathrm{Pu}_{\mathrm{Guo}}{ }^{1}$, Jiaxin Shao ${ }^{2}$, Jiaqi $\mathrm{Tu}^{3}$, Xinhong Xiong* \\ ${ }^{1}$ Department of Mechanical Engineering and Automation, Wuhan University of Technology, Wuhan, Hubei, 430070, China \\ 2 Department of Mechanical Engineering and Automation, Wuhan University of Technology, Wuhan, Hubei, 430070, China \\ ${ }^{3}$ Department of Mechanical Engineering and Automation, Wuhan University of Technology, Wuhan, Hubei, 430070, China
}

\begin{abstract}
This smart storage and washing integrated cabinet based on sine mechanism mainly includes three functional modules: cleaning, storage and transportation. Each part of the organization to cooperate with each other to achieve three basic functions, such as no dead-end dishwashing, tableware storage and according to the instructions to take dishes. Not only that, it also enables additional functions such as drying disinfection and residue filtration. Now that intelligence is rapidly spreading, people's hands in tableware management are free, Let people only participate in the beginning and end of the tableware management chain, and provide greater convenience to people's lives.
\end{abstract}

\section{Work background}

With the improvement of people's economic level, the pursuit of life not only stays at the level of food and clothing, but also looks forward to a more convenient and comfortable lifestyle. Therefore, smart home has become the most popular vocabulary nowadays. The kitchen is an extremely important part of people's family life. Today, with the rapid spread of intelligence, the need for intelligent management of tableware is gradually manifested. After the dishwasher is cleaned, the tableware needs to be separately stored in the cabinet. The dishwasher can only do the intelligent cleaning of the tableware. The ordinary cabinet can only store the tableware. The transfer and retrieval of the tableware in the intermediate process requires people to do it themselves. It makes people need to participate in the entire link of tableware management, and it does not really free people's hands. Therefore, it is particularly important to design a smart cabinet that can clean, store, and take tableware. In this smart cabinet, people only need to participate in the two steps of putting in dirty tableware at the beginning and inputting the type and number of tableware at the end. The rest of the process is completed by the cabinet independently, which will provide people with great convenience.

\section{Work design and introduction}

\subsection{Work design}

Starting from the overall structure, the 2020 aluminum profile forms the basic outer frame of the cabinet through corner connectors. The upper and lower drawers are fixed on the aluminum profile through slide rails. The upper drawer is responsible for the washing, drying and disinfection of tableware, and the lower drawer is responsible for the tableware. For storage, both drawers can be pulled out through sliding rails for easy cleaning and installation. A mechanical claw and a lifting platform are installed on the right side of the cabinet for stacking tableware and transporting it to the surface of the cabinet. The whole system is composed of mechanical structure and control system. The mechanical structure includes a spray module at the top of the upper drawer, a sealing module at the bottom of the upper drawer, and a transfer module located inside the upper and lower drawers and at the right end of the cabinet. The nozzle in the spray module can realize the rectangular movement of the nozzle under the action of the three-bar linkage structure, and complete the cleaning of the tableware according to the best cleaning plan through the data fed back by the sensor. The sealing module completes the back and forth and up and down movement of the sealing plate through the screw rod and the cam, which can complete the sealing environment when the upper drawer is cleaned and transfer the tableware to the lower drawer after the cleaning is completed. The spiral line in the conveyor module can complete the horizontal transmission of the tableware. In addition, the mechanical claw can complete the grabbing and stacking of the tableware with the cooperation of the screw slide table. Finally, the lifting table will transport the stacked tableware under the action of the timing belt. come out. The control system controls the steering and speed of each motor. This system relies on the mechanism design and control design to realize the functions of washing, drying, disinfecting, storage and retrieval of tableware. It is a mechatronics product

\footnotetext{
*Corresponding author: xiongxh@whut.edu.cn
} 
integrating multiple functions.

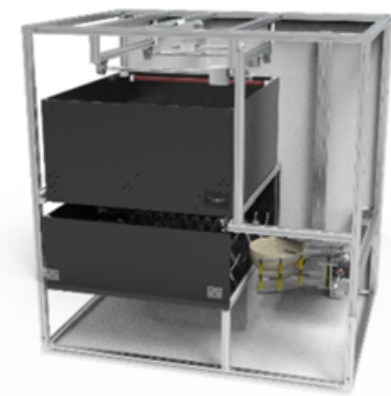

Figure 1 Overall structure diagram of smart storage and washing cabinet

\subsection{Work introduction}

\subsubsection{Spray module}

The spray mechanism includes a sine mechanism, a fixed polished rod, a spray head, and a buffer spring. As shown in Figure 2-2-1, the reciprocating rod of the sine mechanism. According to the theoretical design, the movement trajectory of the nozzle is a rectangle. A spring is applied to this mechanism, and the stiffness coefficient of the spring needs to be selected. Dynamic simulation using Adams found that when the spring stiffness coefficient is small, the movement trajectory of the nozzle is not a rectangle, but a deformed rectangle. Appropriately increasing the stiffness coefficient of the spring finds that the movement trajectory of the nozzle will be approximately a rectangle. As shown in Figure 2-2-2, it is divided into the nozzle movement trajectory diagram when the spring stiffness coefficient $\mathrm{K}=100 \mathrm{~N} / \mathrm{m}$ and the spring stiffness coefficient $K=400 \mathrm{~N} / \mathrm{m}$.

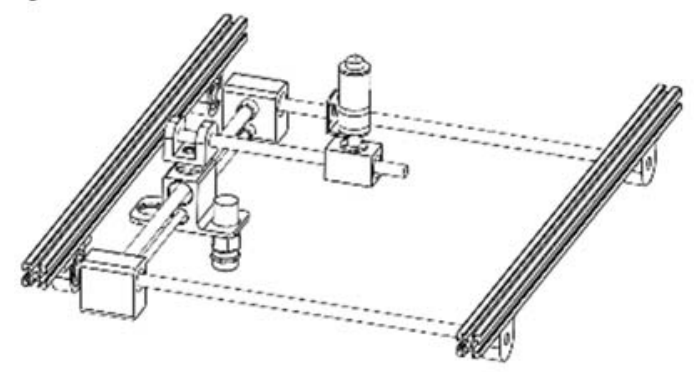

Figure 2 Schematic diagram of spray module
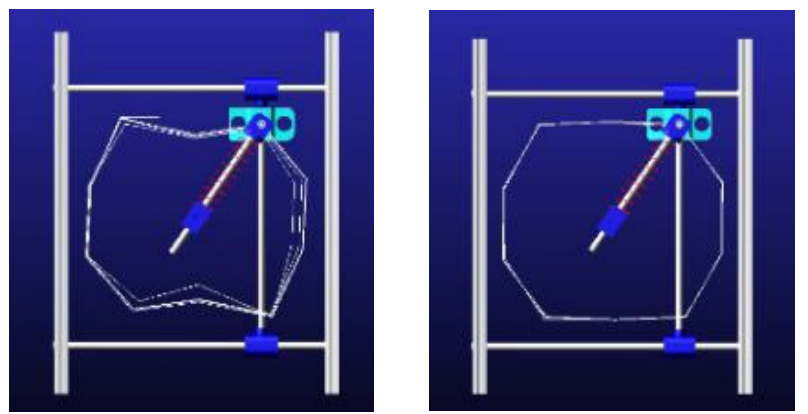

Figure 3 Schematic diagram of nozzle movement track (left $\mathrm{K}=100 \mathrm{~N} / \mathrm{m}$, right $\mathrm{K}=400 \mathrm{~N} / \mathrm{m}$ )

\subsubsection{Spiral bowl transport module}

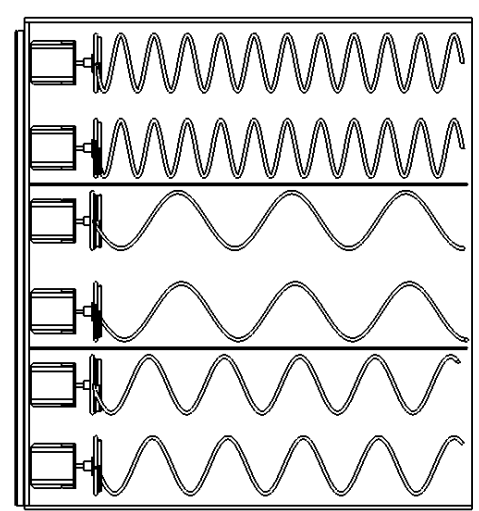

Figure 4 Spiral control

The spiral is one of the cores of the dishwasher, which is used to hold and transport dishes. The spiral wire is controlled by a 42 stepping motor, and the spiral wire is connected to the stepping motor through a self-made coupling. The mechanism has two spiral wires as a group, and the rotation direction is opposite to transport a bowl together. Therefore, the control of the spiral must be synchronized with the two stepping motors in order to make the bowls convey smoothly. The forward, stop, and reverse functions of the stepping motor enable the bowls to be transported in the dishwasher in an orderly manner, which improves the controllability and simplicity. In addition, for different types of bowls, the speed of the spiral is different to ensure the conveying speed and safety of the bowls.

\subsubsection{Sealing module}

The sealing module is composed of a sealing plate, a cam, a connecting plate, a screw rod, and a DC geared motor. As shown in 4.2, through the connecting plate, the screw rod and the screw nut can complete the horizontal movement under the action of the DC geared motor, and the cam installed on the connecting plate can realize the up and down movement of the sealing plate by the steering gear. In addition, a linear slide rail is installed to improve the stability of movement, and a linear bearing is installed to ensure smooth movement. In this way, it is possible to switch between the sealed environment and the open environment of the upper drawer, so that the dishes are washed during sealing and the transfer of the tableware in the upper and lower drawers after washing is completed.

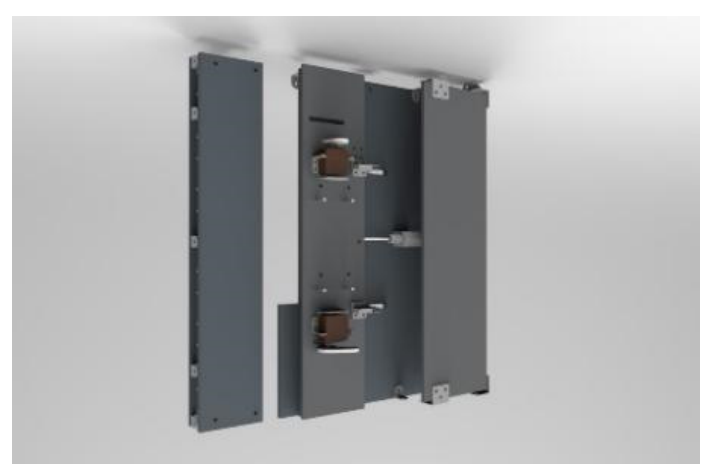

Figure 5 Schematic diagram of sealing module 


\subsubsection{Transport module-mechanical claw}

The mechanical claw transportation part is mainly composed of mechanical claw and screw slide device. The mechanical claw is a clamp-type mechanical claw with symmetrical sides on both sides. The front and back movement is completed by the screw slide table and the opening and meshing of the mechanical claw is completed by the steering gear.

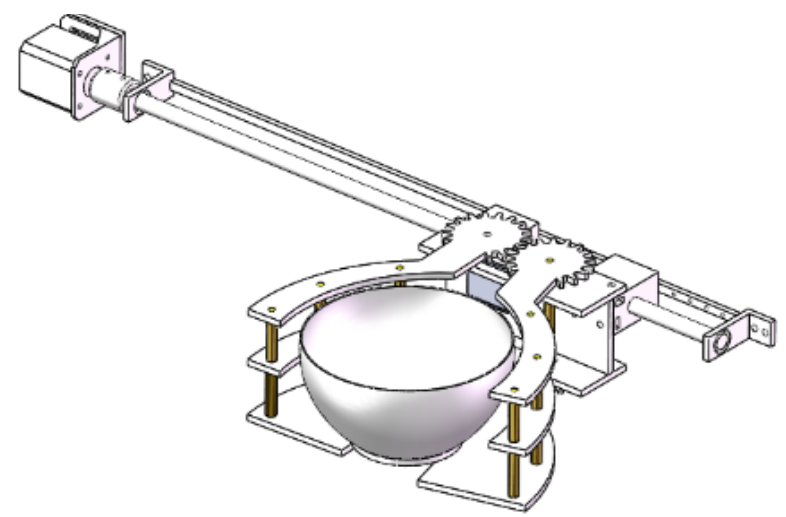

Figure 6 Transportation device

In order to ensure the accuracy of the grip, we design the mechanical gripper according to the size of the cutlery, and the final design of the mechanical claw base plate is shown in the figure:

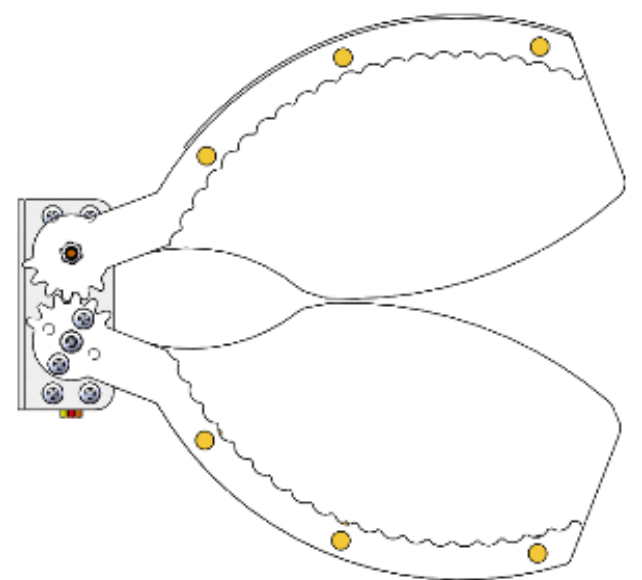

Figure 7 Schematic diagram of mechanical claw bottom plate

\subsubsection{Transport Module-Lifting Table}

The lifting device mainly includes lifting column, lifting platform, motor, timing belt wheel, timing belt, guide wheel, etc. The three-dimensional diagram is as follows:

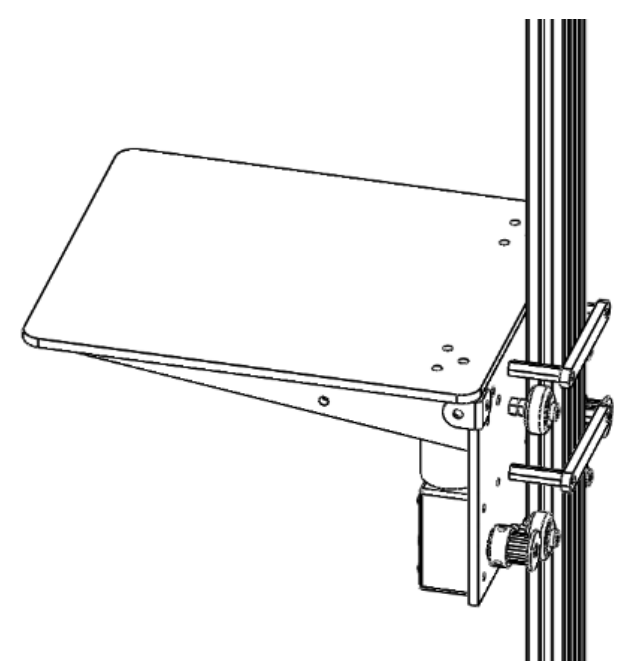

Figure 8 Sketch map of lifting platform

The guide wheels make the lifting platform only move up and down on the lifting column, and the motor drives the synchronous pulley to rotate to realize the lifting movement of the lifting platform.

\subsubsection{Voice interaction module}

The voice interaction module is composed of two major modules: LD3320 voice recognition module and voice broadcast module, as shown in Figure 7-2-6. The voice recognition module is responsible for recognizing the user's instructions, and transmitting the corresponding instructions to the processor. After receiving the signal, the processor issues instructions to take corresponding actions, and sends a signal to the voice broadcast module to answer user-related information. The voice recognition module can recognize all Mandarin Chinese, and can set a firstlevel password and a second-level password. The module uses the LD3320 voice recognition chip, which integrates voice signal acquisition and recognition algorithms. It is equipped with STC11L08XE single-chip microcomputer and communicates with the LD3320 voice module for SP1 communication to realize the function of voice recognition. The module leads out 16 single-chip microcomputer 10 ports, which can complete work alone without supporting other single-chip microcomputers; it can also use the serial port output function of the module to cooperate with other single-chip microcomputers such as Arduino, stm32, etc. The voice broadcast module can be connected to an external source speaker or speaker, the volume can be adjusted intelligently, and feedback information during playback. The module works in two modes: in direct trigger mode, it has 9 direct trigger ports, these 9 ports correspond to $9 \mathrm{MP} 3 \mathrm{~s}$ at the same time, and the trigger mode is to provide low level to the corresponding IO ports. In encoding mode, supply low level to corresponding A10, control the level of 5 IO ports, can play $31 \mathrm{mp} 3-2$ to the 5 th power, can be combined with single-chip microcomputer to complete the operation. 


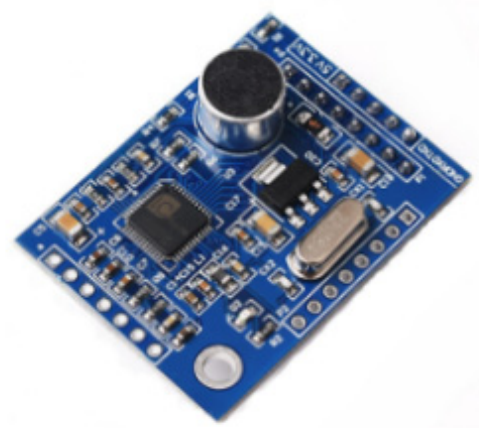

Figure 9 Speech recognition module

\section{Results and Discussion}

With the development of intelligence, smart kitchens will also become a trend, and more convenient, free, and trouble-free will be people's pursuit. This smart cabinet will provide people with great convenience in tableware management and will have a greater competitive advantage in the future. The future is just around the corner.

\section{References}

1. Mao, L., Guo, W., Wu, Z. . (2018) Optimal design of vibration and noise for household dishwashers[J]. Mechanical Design and Manufacturing, (1): 215-218.

2. Xu, B.. (2019) A spray type automatic household cabinet dishwasher[J]. Technology and Innovation, (17): 139-140.

3. Wang, W.. (2004) Mechanical Design Manual. Mechanical Industry Press, Beijing.

4. Wang, X.. (2015) Mechanical Manufacturing Technology. Machinery Industry Press, Beijing. 